\title{
EL PUEBLO DE LA CONFEDERACIÓN SUIZA: SU SIGNIFICACIÓN CONSTITUCIONAL Y COMPOSICIÓN
}

REMEDIO SÁNCHEZ FERRIZ 
SUMARIO

1. EL PUEBLO (CUERPO ELECTORAL) COMO EL ÓRGANO SUPREMO EN TODOS LOS NIVELES DE DECISIÓN POLÍTICA. 1.1 El texto constitucional y la práctica política constante que contrasta con el funcionamiento político en otras democracias. 1.1.1 El texto fundamental suizo es claro tanto en sus concretas expresiones como en su sistemática. 1.1.2 Necesaria clarificación sobre los derechos políticos: su valor institucional como elemento estructural del sistema. 1.2 El pueblo suizo en la práctica política constante y en la adopción de las principales decisiones. 1.2.1 La democracia suiza integra en sí misma las formas conocidas en occidente. 1.2.2 Múltiples formas de participación que condicionan a todo el sistema político. 2. COMPOSICIÓN DEL CUERPO ELECTORAL. DERECHOS DE CIUDADANÍA Y DERECHOS POLÍTICOS. 2.1 Peculiaridades dignas de mención. 2.2 Adquisición de la nacionalidad suiza. 2.3 Adquisición sobrevenida de la nacionalidad: la naturalización tras la Ley de 2014. 2.3.1 Naturalización ordinaria. 2.3.2 Naturalización simplificada o «facilitada». 3. PARA CONCLUIR: EL CÍRCULO CERRADO DE LA VOLUNTAD POPULAR COMO DECISIÓN ÚLTIMA. 


\title{
EL PUEBLO DE LA CONFEDERACIÓN SUIZA: SU SIGNIFICACIÓN CONSTITUCIONAL Y COMPOSICIÓN
}

\author{
REMEDIO SÁNCHEZ FERRIZ* \\ Universitat de Valencia
}

\section{EL PUEBLO (CUERPO ELECTORAL) COMO EL ÓRGANO SUPREMO EN TODOS LOS NIVELES DE DECISIÓN POLÍTICA}

La Constitución suiza es muy clara en la determinación del pueblo como órgano supremo de la Confederación y de sus funciones como tal en los diversos ámbitos de decisión ya desde su mismo inicio, en el Preámbulo ${ }^{1}$. La recurrente afirmación de que estamos ante el primer órgano del Estado no es fruto de interpretación doctrinal o jurisdiccional alguna, sino de la expresa y reiterada previsión constitucional y de la realidad política suiza, intensamente sentida por el pueblo suizo, que la considera un distintivo propio de su particular sistema político, y que se materializa en el constante ejercicio de los derechos políticos en todas las formas que al efecto establece el texto fundamental. Nos referimos a ambos aspectos en unas breves consideraciones.

* Departamento de Derecho Constitucional, Ciencia Política y de la Administración. Facultad de Derecho. Universidad de Valencia. Avenida de los Naranjos, s/n. 46022 Valencia. Email: remedio.sanchez@uv.es

1 «El pueblo suizo y los cantones... otorgan la siguiente constitución...». Desde estas primeras palabras de la Constitución la condición de sujeto constituyente del pueblo (junto a los Cantones) queda fuera de toda duda, sin perjuicio de que a lo largo del texto se irá reafirmando dicha condición que, además, en el caso concreto de Suiza trasciende todo el sistema político y sus principales instituciones. 


\subsection{El texto constitucional y la práctica politica que contrasta con el funcionamiento político en otras democracias}

El pueblo, referente constitucional que legitima la adopción de las grandes decisiones políticas en los Estados democráticos, es, en todos ellos, objeto de invocación en momentos de especial solemnidad y es llamado (como en el caso español e italiano, entre otros) a refrendar no solo la aprobación de la Constitución sino también sus reformas de mayor calado. Pero, junto a ese papel supremo (apartado de la política cotidiana, y reducido generalmente a la función electoral en las democracias representativas como son las dos mencionadas), el pueblo, por más que permanentemente invocado a través de la opinión pública (y, por supuesto, en las campañas electorales), en nuestros países de referencia no se manifiesta cotidianamente ni se relaciona directamente con la función legislativa y la ejecutiva que son las que habrán de mantener vivo el ordenamiento jurídico adecuándolo a la evolución social permanentemente y aplicándolo día a día. $\mathrm{Ni}$ que decir tiene que en la actuación de los dos poderes constituidos mencionados (el legislativo y el ejecutivo) pesa mucho más el juego partidista, y en particular la posición ideológica del partido político que se halle en el poder; y pesa mucho más que la voluntad popular cuya voz, formalmente, solo dispone de vías para dejarse oír en el proceso electoral.

Por ello, el caso suizo es bien distinto; porque la invocación del pueblo que, según la Constitución suiza, es el órgano supremo de la Confederación, no es una simple formulación grandilocuente sino que halla a lo largo de dicho texto fundamental prescripciones muy claras sobre sus competencias reales en el desarrollo de la vida política ya sea en relación con el poder ejecutivo ya con el legislativo e, incluso, con el judicial. Por ello, califica Fleiner ${ }^{2}$ al pueblo suizo en forma aparentemente contradictoria: como poder supremo y, a la vez, como oposición a todo poder constituido.

1.1.1 El texto fundamental suizo es claro tanto en sus concretas expresiones sobre el pueblo como en la sistemática general en que se inserta

Sin perjuicio de la invocación del Preámbulo ya referida, el carácter constituyente del pueblo, junto a los Cantones, queda determinada en el art. 1 de la

${ }^{2}$ Fleiner, Thomas, Misic, Alexander, Töpperwien, Nicole. Swiss Constitutional Law, The Hague, Kluwer Law International, 2005, pág. 60. 
Constitución federal ${ }^{3}$. Y ella misma, acto seguido, establece en el art. 2 como fines de la Confederación justamente la tutela «de la libertad y de los derechos del Pueblo». Aubert subraya el interés y la significación de estas primeras menciones constitucionales y en particular la introducción, novedosa respecto de las Constituciones anteriores, de la expresión «pueblo suizo» en la vigente Constitución de 1999; con tal introducción, que debe subrayarse que fue propuesta por el Consejo de Estados, se quiso sustituir la expresión «pueblos de los cantones» que figuraba en las Constituciones de 1848 y $1874^{4}$.

Explica Aubert tal situación en los textos constitucionales precedentes al ahora vigente, de no mención expresa del pueblo suizo, porque entonces, cuando se redactaron aquellas Constituciones, el pueblo aun no existía jurídicamente sino que progresivamente fue desarrollándose después con tal plenitud que bien merecía recogerse, entonces sí (en 1999) en el texto fundamental y en particular en el art. 1 pues, al insertarlo en dicho artículo, «se demostraba que la Confederación suiza se caracteriza, no solamente por el federalismo sino, también, por la democracia» ${ }^{5}$.

A partir de ahí, son muchas las referencias contenidas en la Constitución en las que la regla general no es otra que el requerimiento del consentimiento popular siempre que se halle en juego la adopción de decisiones importantes.

3 Art. 1 Const.: «El pueblo Suizo y los cantones de Zurich, Berna, Lucerna, Uri, Schwyz, Unterwalden (Alto y bajo), Glaris, Zug, Friburgo, Soleura, Basilea (Ciudad y Campo), Schaffhausen, Appenzell (las dos Rodas), Saint-Gall, Grissones, Argovia, Turgovia, Tesino, Vaud, Valais, Neuchâtel, Ginebra y Jura forman la Confederación Suiza».

${ }^{4}$ Y como tantos otros aspectos constitucionales del país alpino, este no es menos sorprendente para el observador extranjero y, en especial, para quien conoce las permanentes reivindicaciones de algunos territorios españoles que consideran ofensivo (o al menos inoportuno) referirse al pueblo español (tal como recientemente está ocurriendo en Cataluña); por el contrario, en el caso suizo ha de subrayarse que la novedad de la mención del "pueblo suizo» procede de la Cámara que representa a los Cantones y, por consiguiente a los pueblos de los cantones que, según se explica en el texto, fueron sustituidos por el pueblo suizo en el nuevo texto constitucional de 1999.

5 Aubert, Jean-François, y Mahon, Pascal, Petit commentaire de la Constitution fédéral de la Confédération suisse du 18 avril 1999. Zurich, Schulthess, 2003, pág. 16. Resulta curiosa otra precisión del mismo autor que explica cierta incoherencia del verbo «formar» que viene a avalar la reflexión de orden comparado que nos hemos permitido introducir en la nota precedente: según Aubert, la mención de los pueblos de los cantones como estaba prevista en el proyecto no hubiera supuesto demérito alguno para el pueblo suizo pues aquella estaba pensada para referirse a la conformación de la estructura material de la Confederación y por ello se usó el verbo «formar» (según reza el art. 1 Const. «el pueblo suizo y los Cantones.... forman la Confederación». El cambio ya explicado naturalmente se hizo con la pretensión de reforzar la legitimidad de tal configuración confederal pero, lógicamente, concluye Aubert, ahora resulta raro dicho verbo que se cohonesta poco con la idea de legitimidad. 
El Título IV de la Constitución, en el que se regulan los derechos políticos ${ }^{6}$, se encabeza también con la invocación del Pueblo y los Cantones, los dos poderes constituyentes de la Confederación sin cuya intervención no cabe adoptar decisión alguna de trascendencia constitucional. $\mathrm{Y}$ en este mismo sentido, no puede pasar por alto que ello representa una constante que, arrancando de los mismos orígenes de la Confederación, no se ha visto afectada negativamente con el paso del tiempo; por el contrario, debe subrayarse que la evolución histórica del constitucionalismo suizo a partir de la conformación del Estado federal no ha derivado en una debilitación del papel del pueblo sino, al contrario, en su fortalecimiento, que se ha ido manifestando en formas diversas; no solo mediante la progresiva incorporación de instrumentos democráticos que posibilitan la intervención popular cada vez en más instituciones y en formas más diversas, sino también en la clarificación del papel del pueblo en los Cantones.

Piénsese simplemente en el siguiente ejemplo: la exigencia de doble mayoría en decisiones de relevancia constitucional era general en la Constitución de 1848 pero cabía que cada Cantón decidiera el modo de la manifestación de su voluntad popular; sin embargo, a partir de 1874 la voluntad de cada Cantón se manifiesta, necesariamente, a través de la mayoría popular en su propio ámbito. Pero tal fortalecimiento democrático podría ilustrarse con otros hitos de la evolución constitucional. Dando por supuesto que el ámbito comunal es el núcleo originario del modo suizo de concebir y practicar la democracia durante siglos ${ }^{7}$ ). En un posterior momento los hábitos participativos connaturales en los reducidos ámbitos de convivencia comunal se irán formalizando en los niveles de gobierno más amplios enriqueciendo el sistema de democracia esencialmente representativa en que se había organizado Suiza en 1848. En efecto, en dicha Constitución ya se preveía el referéndum obligatorio en materias de reforma constitucional; pero es a partir de entonces cuando se va enriqueciendo y ampliándose progresivamente; en el texto fundamental de 1874 , se introduce el referéndum facultativo en materia legislativa y, en 1891, se introduce la iniciativa popular para

${ }^{6}$ Tras el Capítulo 1. ${ }^{\circ}$, que bajo el epígrafe de Disposiciones generales, contiene dos artículos, el 136 (Derechos políticos) y el 137 con una breve mención de los partidos políticos, el Capítulo $2 .{ }^{\circ}$ se ocupa con detalle de las intervenciones populares a través de la iniciativa y el referéndum. La síntesis de tal Título podemos hallarla en el art. 136: «1. Todos los Suizos y Suizas mayores de 18 años que no estén impedidos por causa de enfermedad o retraso mental, tienen derechos políticos a nivel federal. Todos tienen los mismos deberes y derechos políticos. 2. Podrán tomar parte en la elección del Consejo Nacional y en las demás votaciones federales, así como proponer y firmar iniciativas populares y referéndum en materia federal».

7 Por todos, Es muy clara la síntesis que ofrece Gerotto, Sergio, Suiza. Sobre cómo se gobiernan los suizos. Madrid, CEPC, 2015, pág. 60. Para mayor detalle puede consultarse AuER, Andreas. Le caratteristiche della democrazia diretta locale in Svizzera, en Amministrare, 1999. 
solicitar la reforma parcial de la Constitución mediante 50.000 firmas (que aumentarían a 100.000 , en $1977^{\circ}$ ). En 1921 se añadió la posibilidad de solicitar el referéndum también para los tratados internacionales no denunciables de duración indeterminada (ampliándose en 1977 a otro tipo de tratados), etc., etc. Si a ello añadimos la ampliación en las formas de votación popular que también se han llevado a cabo en los Cantones, no es de extrañar que hoy más de la mitad de las votaciones populares que se producen en el mundo se desarrollen en Suiza.

\subsubsection{Necesaria clarificación sobre los derechos políticos: su valor institucional como elemento estructural del sistema}

Aclaremos de entrada que la Constitución suiza regula y enumera en su Título II los «Derechos fundamentales, derechos cívicos y objetivos sociales». Siendo unánime la doctrina sobre la distinción entre derechos constitucionales y fundamentales ${ }^{9}$, debe llamarse, sin embargo, la atención sobre el hecho de que de estos segundos se ocupan los arts. 7 a 36 que conforman el Cap. I (justamente bajo el epígrafe de «Derechos fundamentales») mientras que precisamente a los derechos sobre los que ahora nos detenemos se refiere el Cap. II bajo el epígrafe de «Ciudadanía y derechos políticos» (arts. 37 a 40) ${ }^{10}$.

La naturaleza, también fundamental, de los derechos políticos queda fuera de toda duda al haber sido garantizados por el propio constituyente en el art. 34 $\mathrm{y}$, por consiguiente, dentro aun de la enumeración de los derechos fundamentales contenidos en el Capítulo I aunque precisamente sean los últimos a los que,

8 La razón del considerable aumento hasta doblar el número de las firmas exigidas en 1977 es coherente con el correspondiente aumento del cuerpo electoral como consecuencia derivada del reconocimiento del derecho de voto a la mujer en 1971, de suerte que, aumentando la exigencia del número de las firmas, sobre mantener con tal aumento el nivel porcentual de la exigencia de firmantes, tal cambio además evitaría una utilización abusiva de dichas instituciones.

9 Entre tantos, Grisel, Etienne, Droits Fondamentaux. Libertés idéales, Berna, Stämpfli Editions, SA, 2008.

10 Entre los artículos de dicho capítulo hemos de subrayar ahora el interés del núm. 39 que, bajo el epígrafe de «Ejercicio de los derechos políticos», establece lo siguiente:

1. La Confederación regulará el ejercicio de los derechos políticos a nivel federal; los cantones regularán estos derechos a nivel cantonal y municipal.

2. Los derechos políticos se ejercen en el lugar de domicilio. La Confederación y los cantones podrán prever excepciones.

3. Nadie puede ejercer derechos políticos en más de un cantón.

4. Los cantones podrán establecer que las personas recién establecidas en su territorio no ejerzan el derecho de voto a nivel cantonal y municipal hasta pasado un plazo máximo de tres meses desde el cambio de domicilio. 
justamente, se va a referir este Capítulo consagrándolos en una regulación genérica como un tipo más de los que constituyen los derechos fundamentales en sentido estricto, para volver a ocuparse de los mismos, ya con mayor detalle y desde el punto de vista práctico que a tales derechos caracteriza, en el Capítulo siguiente tal como acabamos de decir.

Por lo que se refiere al art. 34, bajo el epígrafe de «Derechos políticos», se limita a decir que «los derechos políticos están garantizados y que la garantía de los derechos políticos protege la libre formación de la voluntad y la expresión fiel del voto», reafirmando de este modo el sentido finalista de los mismos en la medida en que se dirigen a manifestar la voluntad del pueblo soberano. No cabe dudar, por consiguiente, de la consideración de los derechos políticos como un grupo más de los derechos fundamentales aunque la convicción de que conforman un grupo especial explica que su regulación específica se difiera a otras ubicaciones del texto fundamental, siendo la primera de ellas (en lo que al ámbito federal se refiere) el inmediato Capítulo II del mismo Título II.

En efecto, los derechos políticos a que se refiere el último artículo del Capítulo I, única y expresamente dedicado a los derechos fundamentales, si de una parte quedan referidos en la propia Constitución a la garantía que a sus titulares asiste, de otra parte debe subrayarse que tal garantía se define con carácter institucional en la medida en que los derechos políticos son garantizados con el fin de que la voluntad popular y su transformación en voluntad estatal se conforme libremente y libremente se manifieste a través del voto. Naturalmente, tal como ya ha quedado aludido, el ejercicio del voto adopta en Suiza manifestaciones bien distintas (que cabe sintetizar con los tres verbos: votar, firmar y ratificar) y no solamente las dirigidas a la designación de los representantes a la que estamos acostumbrados en las democracias puramente (o casi) representativas. Ello justifica las diversas ubicaciones de la regulación constitucional por razones sistemáticas.

Todo lo hasta aquí afirmado no es sino la consecuencia lógica de la naturaleza institucional y estructural de los derechos políticos que, sin desmentir su naturaleza de derechos subjetivos fundamentales, acentúa de entre sus elementos constitutivos el de su ejercicio real y su finalidad (capaz de ejercer permanente influencia en toda actuación pública y en la organización y funcionamiento de los órganos estatales, cantonales y municipales) respecto de otros elementos como titularidad, ámbito, límites o defensa que, sin quedar desatendidos, sin embargo se contemplan por el ordenamiento en forma «estática» (así, previsiones normativas como la referida a la especial composición de la sala del Tribunal Federal cuando ha de conocer de ellos que sólo se actúan en forma ocasional, o en cuanto a titularidad, la normativa sobre adquisición de la ciudadanía); mientras que el valor institucio- 
nal y estructural, a diferencia de lo que ocurre en los países de nuestro entorno, no solo se manifiesta en el caso suizo en forma dinámica por ser el derecho de ejercicio por excelencia, sino por el hecho de representar una permanente presencia de la ciudadanía en la adopción y el control de las decisiones políticas lo que comporta modificaciones en el comportamiento y estructura de las instituciones.

No en vano, los derechos políticos son instrumentos a través de los cuales el cuerpo electoral, entendido como órgano supremo del Estado, ejerce sus funciones. El ejemplo que, sobre la adquisición de la ciudadanía podremos ver en el último epígrafe de este trabajo, nos ofrece un claro contraste entre la normativa de aplicación y la realidad de unos derechos políticos activos (y en actitud «activista») capaces de enfrentarse a las autoridades aun cuando estas tratan de imponer el Estado de Derecho.

Y siendo ello así, ha de subrayarse su carácter transversal; pues no se limita a determinar las estructuras federales y cantonales ${ }^{11}$ sino que también conforma la naturaleza de los municipios suizos y demás entidades conocidas como comunas. El ejercicio de los derechos políticos a nivel local conforma la naturaleza y estructura municipal o, dicho de otro modo, los municipios son lo que son por ser el espacio natural de expresión de la ciudadanía y lo peculiar de su estructura varía de unos a otros por variar justamente sus modos de expresión.

Y llama la atención que en el nivel federal también se deje sentir la permanente posibilidad de que el cuerpo electoral se exprese rectificando lo decidido por los poderes constituidos y ello en formas diversas: en primer lugar, condicionando la agenda de tales poderes, en segundo lugar, forzando acuerdos y consensos que tal vez de otro modo no se producirían; por último, generando una red de contactos institucionales a modo de "para administración», que mitiga o compensa en el nivel federal la falta (lógica) de arraigo de institutos que sí tienen presencia permanente en las decisiones comunales (p. ej. Asambleas) o cantonales (iniciativa popular legislativa ${ }^{12}$ ) pero que no podría ejercerse en ámbi-

${ }^{11}$ Sobre los efectos que en las estructuras institucionales genera el permanente ejercicio de los derechos políticos, en ocasiones de modo disfuncional y, por consiguiente, criticado en ámbitos doctrinales, entre otros, Papadopoulos Yannis. Les mécanismes du vote référendaire en Suisse: l'impact de l'offre politique. In: Revue française de sociologie. 1996, 37-1. pp. 5-35. Disponible en red:

http://www.persee.fr/web/revues/home/prescript/article/rfsoc_0035-2969_1996_ num_37_1_7078. Del mismo autor, junto a otros, puede consultarse: Débat: Déliberation et action publique, en Swiss Political Science Review 10(4), 2004, págs.. 147-210 También, Joye, Dominique La democrazia locale e diretta in Svizzera, en Amministrare, 1999, pp. 169-189.

12 No han cuajado los intentos de extender la iniciativa popular a la competencia de la legislación ordinaria en el ámbito federal en el que sigue siendo posible tal iniciativa solamente para las reformas constitucionales. 
tos muy poblados. La consolidación del procedimiento de consultas ${ }^{13}$ y su reconocimiento formal en la Constitución vigente se enmarcaría en estas consideraciones sobre los efectos institucionales del ejercicio de los derechos políticos.

\subsection{El pueblo suizo en la práctica política constante y en la adopción de las principales decisiones}

\subsubsection{La democracia suiza integra en sí misma las formas conocidas en occidente}

Es también Aubert ${ }^{14}$ quien nos ofrece la mejor y más clarificadora síntesis de las experiencias democráticas conocidas en el mundo occidental y de la significación de los dos adjetivos más frecuentemente usados cuando escribimos sobre la democracia suiza: «directa» y/o «semidirecta».

Así, nos recuerda que existen en las experiencias democráticas tres niveles: por el primero, el mínimo, el cuerpo electoral (o fracción del pueblo con capacidad para ejercer las funciones en nombre de todo él) elige a quienes les van a representar ejerciendo funciones legislativas (si se trata de elegir los miembros del Parlamento) o ejecutivas (si se eligen los gobiernos, como en el ámbito cantonal), razón por la cual la democracia en este primer nivel se llama representativa; si además de tales funciones electivas, se le reconoce la capacidad de ratificar o no las normas aprobadas por los representantes a través del referéndum, se estaría en un segundo nivel que aún se vería fortalecido si el referéndum pudiera ser pedido por el propio cuerpo electoral sin esperar a la convocatoria de las autoridades, como también ocurre en el caso del derecho de iniciativa popular, situación que se suele calificar de democracia directa aunque en realidad se trata de una democracia semidirecta en la medida que la directa en sentido estricto es la que se ejerce directamente por el pueblo reunido en asamblea.

Pues bien, los tres niveles descritos concurren en el ordenamiento suizo a modo de experiencia única en el mundo pero con una peculiaridad digna de subrayarse tanto desde la perspectiva de las previsiones institucionales del ordenamiento democrático como desde las convicciones culturales de los suizos: se trata de la relación que existe entre los tres tipos de democracia que en el suelo

13 Cfr. SÁnchez Ferriz, Remedio. Un mecanismo de integración federal y ciudadana: las consultas «prenormativas» del ordenamiento constitucional suizo, en Teoría y Realidad Constitucional, núm. 36, 2015, pp. 353-376.

14 Aubert, Jean-François, y Mahon, Pascal, Petit commentaire de la Constitution fédéral de la Confédération suisse du 18 avril 1999. Zurich, Schulthess, 2003, pág. 1062. 
suizo concurren; pues priman entre ellos en el orden inverso en que se han descrito; es decir, que, derivando el poder popular de abajo a arriba (o de las comunas al Cantón y de este a la Confederación), el derecho de voto carece de la relevancia de que gozan los derechos políticos a través de los cuales el cuerpo electoral puede manifestarse en cualquier momento; y lo mismo cabe afirmar sobre los sistemas de asamblea allí donde se practican.

Ello es así; de suerte que, sea institucional o culturalmente, e incluso desde el punto de vista de la frecuencia, cabría decir que ante todo prima la democracia directa, después la semidirecta y, por último, la representativa. Se ha llegado a afirmar que «la democracia directa es la razón de ser de un Estado en el que la misma denominación oficial - «Confederación» suiza- indica que se trata de una comunidad casi jurada de ciudadanos demócratas...» ${ }^{15}$. Naturalmente los ciudadanos requieren de instrumentos con los que ejercer su poder supremo y manifestar permanentemente su compromiso con el sistema. Tales son los derechos políticos ${ }^{16}$.

\subsubsection{Múltiples formas de participación que condicionan a todo el sistema político}

La realidad no solo se manifiesta en los más conocidos instrumentos democráticos a que se acaba de aludir con el nombre de derechos políticos ${ }^{17}$, sino en otras muchas peculiaridades que resultan extrañas al observador que los estudia desde perspectivas constitucionales diferentes; hasta el punto de que en Suiza se

15 Sigg, Oswald La Svizzera política. Zurigo, Pro Helvetia, 1996, pág 19.

16 De los mismos me he ocupado con detenimiento en una publicación de próxima aparición, coordinada por GerotTo, Sergio. La complejidad de su estudio y la reducida dimensión de este trabajo me obligan a llevar a cabo ahora una referencia muy general (la mínima imprescindible) a los mismos. A mi juicio la obra más completa para conocer el ejercicio real de los mismos en todos los ámbitos territoriales es la de GRISEL, Etienne, Initiative et referéndum populaires. Traité de democratie semi-directe en droit Suisse. Bern, Stämpfli Editions, 2004

${ }^{17}$ Las funciones o competencias del cuerpo electoral (compuesto por los nacionales de origen y los naturalizados) son las formas por las que se manifiesta la voluntad popular que, a diferencia de los países democráticos en que se expresa mediante la emisión del voto, en Suiza adquiere una triple manifestación que cabe sintetizar en las tres acciones de elegir, votar, firmar (cfr. AUER, Andreas, MaLinverni, Giorgio y HotTelier, Michel Droit, constitutionnel suisse. Berne, Stämpfli Editions, SA, 2006, pág. 25). Es decir, el pueblo se manifiesta optando en las elecciones entre las diversas ofertas políticas para elegir a los miembros de los cuerpos representativos, optando entre las opciones que se le someten en referéndum, y firmando ya sea propuestas de iniciativa, ya sea peticiones de referéndum cuando es de carácter facultativo, ya sea alguna petición colectiva. A todas estas formas que la Constitución reconoce como diferentes modos de expresarse la voluntad (participativa) popular, la doctrina las reconduce esencialmente a su regulación de los derechos políticos. 
han consolidado como caso único en el mundo constitucional democrático. Aunque sea con brevedad, la excepcionalidad del régimen suizo vinculada al papel del pueblo, puede ilustrarse con las siguientes consideraciones:

a) La tradicional ausencia de control de constitucionalidad en el ordenamiento suizo ${ }^{18}$ responde a la incoherencia que supondría que un órgano estatal, por más que fuera un Tribunal Constitucional o Supremo con tal función, pudiera revisar una decisión en la que hubiera participado directamente el cuerpo electoral que es quien tiene la última palabra, cosa harto frecuente, y por lo demás segura respecto de toda decisión de relevancia constitucional por propio mandato de la Constitución de la Confederación ${ }^{19}$.

b) Tal vez más decisiva aún es la peculiaridad que se observa en cierto forcejeo entre los órganos constituidos y el cuerpo electoral a la hora de que prevalezcan principios elementales del Estado de Derecho cuales son el derecho de recurso y la protección de la privacidad que el Tribunal Federal ha debido invocar y proteger abiertamente en el caso del reconocimiento de las naturalizaciones mediante el voto popular sobre la que después volveremos.

c) De no menor transcendencia es la prevalencia de la voluntad popular en cuestiones de orden internacional que explican la lenta y tardía aproximación a la ONU hasta su definitiva integración, así como las difíciles relaciones mantenidas con la Unión Europea a través de Pactos bilaterales no exentos de hallarse bajo la espada de Damocles de las iniciativas populares como recientemente se ha puesto de relieve con el resultado del referéndum que obliga a las autoridades federales a llevar a cabo restricciones mediante el establecimiento de cupos para la inmigración (iniciativa votada en febrero 2014) ${ }^{20}$.

No cabe duda de la gravedad de la cuestión y la eventual contradicción que el ejercicio democrático puede comportar frente al Derecho interna-

18 Gerotto, Sergio, Suiza. Sobre cómo se gobiernan..., ya cit, págs. 127 y ss.

19 Es cierto que recientemente han ido cambiando las estructuras (desde su última reforma completa en marzo de 2000, que ha ido entrando en vigor en los años sucesivos a medida que se fue desarrollando) y funciones jurisdiccionales (a través del «control de la convencionalidad» que la aplicación del CEDH ha supuesto) pero sigue sin existir hoy el control abstracto de las leyes federales.

${ }^{20}$ El 9 de febrero de 2014 el pueblo suizo voto la iniciativa popular «Contra la inmigración de masa» imprimiendo un giro importante a la política migratoria. Ahora el Consejo federal y el Parlamento deberán establecer, en tres años, un nuevo sistema de admisiones con el que pueda Suiza regular y limitar la inmigración en atención a la salvaguardia de los intereses de la economía. 
cional y a los derechos humanos ${ }^{21}$. Sin embargo, tampoco cabe desconocer que, precisamente la atenuación de la ausencia de control de constitucionalidad a que nos acabamos de referir se ha producido por vía de las obligaciones internacionales derivadas de la ratificación del Convenio europeo.

d) Tal vez la superioridad de los poderes del cuerpo electoral pueda explicar también el retraso (considerable respecto de los países del entorno) con que el sufragio universal ha alcanzado a las mujeres en Suiza pues tal decisión en última instancia dependía del entonces cuerpo electoral conformado solamente por los hombres.

e) Esta misma idea de soberanía residenciada en el pueblo, y ejercitada por él directamente, se aplica del mismo modo en los otros dos niveles territoriales. Así, no cabe duda de que la soberanía cantonal (consagrada en el art. 3 de la Constitución federal) se hace recaer en el pueblo entendido como el conjunto de todos los ciudadanos del Cantón. Y ello queda perfectamente establecido en la misma Constitución de la Confederación cuyo art. 51 establece un marco constitucional mínimo a partir del cual los Cantones (su órgano constituyente) redactan su propia Constitución en ejercicio de su autonomía constitucional ${ }^{22}$ a condición que tanto ella como sus reformas dependan de la voluntad popular.

Así pues, tal marco mínimo tiene como único referente al pueblo cantonal que es mencionado por el artículo 51 de la Constitución desde diversas perspectivas pero siempre con la misma acepción de órgano supremo del Cantón referida a su propio cuerpo electoral ${ }^{23}$. En efecto, en el primer párrafo no solo exige el carácter democrático a toda constitución cantonal sino que precisa en forma expresa que es el pueblo quien la debe aprobar; pero la segunda parte de este primer párrafo es determinante pues, a reglón seguido, tras afirmar que ha de ser

${ }^{21}$ Por todos, Mahon, Pascal. Droit constitutionnel. 3 ed. Helbing Lichtenhahn, Vol. I, 2015, págs. 185 ss

22 Martenet, Vincent. L'Autonomie constitutionnel des Cantons. Bâle-Geneve, Helbing\&Lichtenhahn, 1999, págs. 101ss.

${ }_{23}$ Dispone textualmente el art. 51 de la Const., con el epígrafe de «Constituciones cantonales», lo siguiente:

1. Cada cantón se dotará de una Constitución democrática. Ésta necesitará la aprobación de la población y deberá poder ser reformada si así lo solicitase la mayoría del electorado.

2. Las Constituciones cantonales tendrán que estar garantizadas por la Confederación. Ésta podrá garantizarlas si no son contrarias al Derecho Federal. 
aprobada por el pueblo, añade que «será revisada siempre que la mayoría ${ }^{24}$ del pueblo lo pida».

De ahí, el interés del epígrafe del Título IV que invoca al pueblo y los cantones, menciones que no van referidas a las organizaciones territoriales (y sus órganos de gobierno con las respectivas competencias) sino órganos de la Confederación como a su vez lo es todo el pueblo de la misma con carácter de supremo ${ }^{25}$.

f) Por último, y antes de entrar en alguna consideración sobre la composición del cuerpo electoral aun hemos de recordar algo muy significativo: los suizos se sienten suizos siendo como son muy diferentes entre sí tanto en la religión, como en la lengua y culturas diferentes. Y, sin embargo no se pone en duda su pervivencia como pueblo que se atribuye a la decidida voluntad de permanecer juntos: «....un pueblo que no se mantiene unido por afinidad de lengua, cultura u otra, sino por la (no) simple voluntad de permanecer unidos» ${ }^{26}$.

Tal realidad, de fragmentación sociocultural, unida a la fragmentación política, hace más imprevisibles las posibilidades de actuación de las diversas fracciones del cuerpo electoral, fundamentalmente en lo que se refiere al ejercicio de la iniciativa y del referéndum facultativo. De tal suerte que nos hallamos ante un «pueblo» que en cada ocasión y en cada tema a tratar puede manifestarse en formas y sentidos no siempre predecibles. Ello sin olvidar que a las culturas tradicionales en Suiza se suman hoy nuevos grupos sociales derivados de la inmigración ${ }^{27}$ cuya integración por la necesaria aceptación de los valores consti-

${ }^{24}$ Sobre la discusión doctrinal de lo que deba entenderse por mayoría (de votos válidos emitidos o de electores inscritos), Martenet, Vincent. L'Autonomie constitutionnel ..., ídem, pág. 105.

${ }^{25}$ La naturaleza democrática de estas partes constitutivas y constituyentes de la Confederación no solo se deduce del art. 51 mencionado. Precisa Aubert (Aubert, Jean-François y MaHon, Pascal, Petit commentaire de la Constitution ... ya cit., pág. 1062) que la garantía de los derechos políticos que se consagra en el art. 34 de la Constitución se dirige a su ejercicio en todos los ámbitos territoriales de suerte que viene a representar la protección federal de los derechos políticos que los Cantones reconocen a sus ciudadanos. En esta misma línea de pensamiento hay que recordar el «fin» de la Confederación que, como hemos señalado ya, no es otro que la tutela de los derechos del pueblo de conformidad con el art. 2 Const.

${ }^{26}$ Gerottro, Sergio, Suiza. Sobre cómo se gobiernan... ya cit., pág. 20.

${ }^{27}$ Cfr. Junto a la tradicional pluriculturalidad y su multilingüismo oficial, se cuentan importantes colonias de extranjeros, lo que apenas constituye particularidad digna de subrayarse por ser una realidad extendida en toda Europa (en este sentido, MAHON, Droit...ya cit, pág. 39, enumera los grupos más significativos de residentes: italianos, alemanes, portugueses, etc.). Respecto de las dificultades de integración, en especial de las colonias musulmanas, Previtali, Adriano. El derecho Constitucional suizo entre el multiculturalismo, multijuridicismo e integración, en 
tucionales suizos sigue constituyendo uno de los temas más debatidos como vamos a ver.

Así pues, solo la composición de la fracción «activa» del pueblo (entre el $60 \%$ y el $75 \%$ de la población suiza constituye el cuerpo electoral) es el elemento previsible y determinado en cada caso al hallarse perfectamente regulada por la Constitución, las leyes federales y las cantonales. A partir de tal conformación reglada, y prefijada en los censos decenales, se lleva a cabo el cómputo de la participación o del abstencionismo, así como el reparto de escaños en la elección del Consejo Nacional o cámara popular. Más allá de estas cifras, las decisiones pueden variar en función de intereses, circunstancias y elementos culturales de muy diversa naturaleza. Me limito en esta ocasión a exponer uno de tales elementos previsibles, el de la composición del cuerpo electoral en el que también hallaremos alguna de las peculiaridades ya aludidas sobre la participación popular en todo tipo de decisiones y sobre los efectos que ello tiene en el sistema.

\section{COMPOSICIÓN DEL CUERPO ELECTORAL. DERECHOS DE CIUDADANÍA Y SU DEPENDENCIA DE LOS DERECHOS POLÍTICOS}

La selección, o modo de extracción, de la fracción del pueblo que, conocida como cuerpo electoral, lleva a cabo las funciones que la Constitución le asigna como órgano supremo de la Confederación, no dista mucho de la conocida en los ordenamientos democráticos. El concepto de nacionalidad, su significación como estrecho vínculo entre los ciudadanos y su Estado, y los derechos y deberes ${ }^{28}$ que de este especial status derivan es, en términos generales, el propio de todo Estado de Derecho. Sin embargo, también en este aspecto existe en el caso suizo alguna peculiaridad digna de recordarse.

\footnotetext{
Anuario de Derecho Penal, 2010. Disponible en red: http://perso.unifr.ch/derechopenal/assets/ files/anuario/an_2012_03.pdf

${ }^{28}$ Mahon (en Aubert, Jean-François, y Mahon, Pascal, Petit commentaire de la Constitution ... ya cit., pág. 333) puntualiza que fue justamente esta condición subjetiva, vinculada a la personalidad de los nacionales, la que, durante la deliberación de la reforma, movió a las Cámaras a cambiar la ubicación constitucional de estos preceptos que en el proyecto gubernamental figuraban en el primer Capítulo del Título III (referido a las relaciones entre Confederación y Cantones), al Título II (referido a derechos, ciudadanía y fines sociales); pues más que a la distribución competencial y a las relaciones entre la Confederación y los Cantones (que también) afectan directamente al estatuto de las personas con relación al Estado.
} 


\subsection{Peculiaridades dignas de mención}

En primer lugar, y enlazando con lo que se acaba de afirmar en el epígrafe anterior, no cabe desconocer que en Suiza el vínculo que en los países de nuestro entorno se establece con el Estado-Nación no tiene la estructura vertical propia de la unidad nacional sino que, al contrario, deriva de una realidad multicultural que se entreteje en multitud de alianzas en las que concurre la voluntad de configurar el Estado y permanecer en él. Lo que no es óbice para que el ordenamiento jurídico establezca las reglas jurídicas al uso: que el cuerpo electoral se conforme con los nacionales mayores de edad, a los que no se les haya privado de sus derechos políticos por sentencia judicial firme; y lo mismo cabe decir sobre que dicha nacionalidad pueda ser de origen desde el nacimiento o adquirida a través del procedimiento y con los requisitos al efecto establecidos y que vamos a ver.

Sin poder entrar ahora en la exposición detallada de todo ello y siendo en lo básico coincidente con los ordenamientos democráticos, como ya he dicho, no puede dejar de mencionarse lo que le caracteriza en virtud de los fundamentos propios de este régimen: federalismo multicultural, con «fuerte dosis de democracia directa»... ${ }^{29}$. En tal sentido, no ha de extrañar que la Confederación comparta las competencias con los Cantones a la hora de regular tanto los diversos aspectos de la normativa sobre nacionalidad como el ejercicio de los derechos políticos a través de los cuales se desempeñan las funciones propias de la ciudadanía.

Así, la Confederación es competente para regular la adquisición y pérdida de la nacionalidad y de los derechos de ciudadanía (art. 38 Const.) lo que se lleva a cabo a través de la Ley federal de ciudadanía o ley de nacionalidad a la que me referiré; pero en lo que se refiere a la adquisición de la misma por naturalización ordinaria tanto los Cantones como los municipios tienen competencias aunque, lógicamente, las eventuales competencias de estos últimos dependerán de que se las reconozca el propio Cantón.

Tampoco, por consiguiente, extrañará que de ello derive una serie de consecuencias como son las siguientes:

7) Que la Constitución federal (art. 37.1) haga depender la nacionalidad federal de su previo reconocimiento en el Cantón y la comuna política o municipio correspondiente, lo que en el caso suizo no se limita a la lógica de la adscripción territorial que hallaríamos en otros países, sino que tal reconocimiento «local» se somete a regulaciones diversas en la medida en que tales entidades territoriales tienen libertad de regulación en el

29 Mahon, Pascal. Droit constitutionnel... ya cit., pág. 153. 
marco establecido por la Constitución; los procesos de naturalización constituyen un buen ejemplo que veremos de inmediato.

8) La variedad de instrumentos de participación política según el territorio en que se hallen los ciudadanos, por cuanto se anudan al reconocimiento de la ciudadanía en cada Cantón concreto y, a veces incluso, en cada municipio;

9) Introducción, a través de tales competencias cantonales, de alguna diferencia normativa que puede llegar a afectar a la regla general de pertenencia al cuerpo electoral como es la de la edad que se ha rebajado hasta 16 años en Glaris; o la posibilidad de ejercicio de los derechos políticos por parte de los extranjeros ${ }^{30}$ (rompiendo, así, la regla de su reconocimiento a solo los ciudadanos como se dispone en el ámbito federal) ${ }^{31}$.

10) Pero, sin duda, la principal particularidad derivada del sistema federal suizo y, muy especialmente en este caso, de la concepción de una soberanía popular que se lleva a la vida política práctica a través de la participación real en decisiones relevantes, es la referida al sistema de naturalización o adquisición sobrevenida de la nacionalidad en aquellos casos en que las decisiones de las autoridades competentes o los principios generales del ordenamiento podrían quedar contrapuestos a las manifestadas por el cuerpo electoral.

\subsection{Adquisición de la nacionalidad suiza}

La ciudadanía suiza es un bien precioso que se hereda o se conquista con mucho esfuerzo ${ }^{32}$. En efecto, la nacionalidad se adquiere ordinariamente de conformidad con las previsiones del derecho de familia en el que se han operado los cambios propios de la democratización y progresiva extensión del principio

30 Cfr. Mahon, Pascal. Droit constitutionnel... ya cit., págs. 161-162.

31 Si bien la cuestión se ha debatido en 22 Cantones, solo una minoría de ellos han aceptado tal inclusión: Por leyes cantonales, y aunque en formas diferentes, han decidido incorporar a los extranjeros a los derechos de ciudadanía Friburgo, Ginebra, Jura, Neuchâtel y Vaud. Este último, junto con Friburgo, conceden el voto tanto activo como pasivo a nivel municipal (no así Ginebra que lo limita al sufragio activo). Jura reconoce el voto y también la accesibilidad a algunas funciones públicas pero solo a nivel municipal mientras que en el nivel cantonal permite el voto con restricciones. En Neuchâtel puede ejercerse el sufragio activo y pasivo en el ámbito municipal y solo el activo en el cantonal. El grupo de Cantones germánicos prefiere, sin embargo dejar este tipo de decisiones a los propios municipios.

32 Gerotto, Sergio, Suiza. Sobre cómo..., ya cit., pág. 111. 
de igualdad (principalmente por su aplicación a la mujer) como también ha ocurrido en nuestro entorno europeo. La aplicación del principio de igualdad ha llevado a modificaciones muy importantes en la materia desde la última década del siglo XX. Así, la situación jurídica de la mujer ha dejado de depender de la de su marido, adquiriendo plena individualidad que le permite tener su propio status civitatis ${ }^{33}$.

La nueva Ley de ciudadanía de 20 de junio de 2014 (LCit), como la de 1952 que ha sido reformada por aquélla totalmente, dedica su Título I a la adquisición y pérdida de la ciudadanía por ley (básicamente por filiación). En la medida en que dicha ley de 1952 ya había sido reformada en múltiples ocasiones, las decisiones que había ido adoptando el TF (al ir reconociendo diversas situaciones de filiación y de convivencia de hecho antes no reconocidas) ya se habían ido incorporando a la vieja Ley por lo que apenas existen novedades (por no haber requerido de nuevos cambios) en este Título I de la Ley de 2014.

En cambio, sí existen notables diferencias en el Titulo II de la nueva Ley (referido a la naturalización) y ello por dos razones: la primera es precisamente la derivada, de la triple intervención en el proceso de naturalización de los tres niveles de la Administración (federal, cantonal y municipal) que exigían una más eficaz coordinación entre las fases y procedimientos de la Confederación y los de los entes territoriales ${ }^{34}$; la segunda, derivada de las recientes peticiones populares en el sentido de que se revisaran tanto la Ley de extranjería como la de Asilo para introducir en ellas mayores exigencias de integración de los extranjeros. Siendo así, era obvio que se debía modificar la Ley de ciudadanía para adecuarla a la nueva normativa concordante tras sus respectivas reformas.

33 Piénsese que antes una mujer perdía la nacionalidad por casarse con un extranjero.... En el caso de la esposa extranjera que adquirió la nacionalidad por matrimonio con un suizo, con la disolución del matrimonio la perdía, como también en el caso de que el esposo hubiera renunciado a la nacionalidad suiza... Situaciones todas que se vieron modificadas en el sentido igualitario mencionado a partir de enero de 2006 (fecha en la que entró en vigor la reforma a la Ley de Ciudadanía Suiza); naturalmente, quienes se vieron afectadas por la legislación anterior gozan ahora de facilidades para su recuperación si demuestran que mantienen lazos estrechos con Suiza. Tales casos se insertan ahora en los supuestos de lo que enseguida vamos a conocer como proceso facilitado o simplificado de naturalización.

${ }^{34}$ El Mensaje Consejo Federal del (de 4 de marzo de 2011) sobre la Ley de Ciudadanía insiste en tal finalidad tendente a la simplificación y la homogeneización de los procedimientos. En él se pone de relieve la delicada situación que se generaba cuando la Confederación, ante el cumplimiento de los requisitos legalmente exigidos se encontraba en el deber de aceptar la naturalización, al tiempo que se manifestaban en contra el Cantón y/o el municipio; ello se ha tratado de resolver ahora designando una autoridad cantonal que reciba las solicitudes de naturalización y estableciendo que solamente se transfiera el expediente a la Confederación cuando ya exista aceptación de la solicitud por el Cantón y el municipio. 


\subsection{Adquisición sobrevenida de la nacionalidad: la naturalización tras la Ley de 2014}

La naturalización, o proceso por el que un extranjero logra la nacionalidad suiza, tiene varias manifestaciones: en lo que se refiere a la dificultad mayor o menor del procedimiento, se distingue entre la ordinaria (arts. 9-19 de la LCit de 2014), y la simplificada (arts 20-25 de la misma Ley).

\subsubsection{Naturalización ordinaria}

En primer lugar, la que se considera ordinaria comporta un proceso muy complejo que se halla regulado por los Cantones a partir de la previsión constitucional del art. $38.2^{35}$ y del marco constitucional en el que habrá de considerarse de modo especial el respeto a los derechos fundamentales. Pero al tratarse de una competencia concurrente con la Confederación (piénsese que la propia Constitución impone el deber de que la regulación federal sea de «mínimos»), son dos los momentos que hasta ahora cabía distinguir:

1) En la primera fase o momento procesal el extranjero ha de obtener de la Confederación la autorización en los términos del art. 12.2, (de la Ley del $\left.1952^{36}\right)$ : «La naturalización es válida solo si el Servicio federal competente (que en realidad es la Secretaria de Estado para la Inmigración) ha concedido una autorización» que será para tres años aunque renovables pero con validez en un único Cantón (art. 13) ${ }^{37}$.

2) El procedimiento a seguir en lo que propiamente sea el reconocimiento de la naturalización es el segundo momento de la tramitación para la obtención de la misma que, sin embargo, no tiene un desarrollo uniforme en la medida en que compete a cada Cantón su regulación, requisitos, y condiciones según el art. 15a de la Ley de aplicación que, además, en su pfo. 2 establece la posibilidad de que, sobre la resolución de tal pro-

35 En el que se dispone que la Confederación dictará disposiciones mínimas sobre la naturalización de los extranjeros por parte los cantones y otorga la autorización de naturalización.

36 Aún vigente a la espera del desarrollo reglamentario de la de 2014.

37 Sin embargo, ello no comportaba, ni comporta, derecho alguno ni a la renovación ni a obtener la naturalización; en realidad se trataba de una tramitación que es una condición sine qua non para en su día poder pedir la naturalización pero no forma parte en realidad del proceso propiamente dicho de esta. Es más, cabría ser revocada si se tuviera conocimiento de nuevos datos que, de haberse conocido en el momento de su concesión, no se hubiera obtenido la misma (según los requisitos que la Oficina mencionada ha de confirmar en los términos del art. 14) y, en cualquier caso, no podía solicitarse hasta pasados 12 años de su residencia en Suiza debiendo tres de ellos ser inmediatamente previos a la petición. 
ceso, se pronuncie el cuerpo electoral del municipio reunido en Asamblea, lo que se justifica por la doble y automática adquisición de la ciudadanía en el municipio y en el Cantón por mandato del art. 38 Const., tal como ya quedo dicho en el epígrafe precedente. Pues bien, siendo ello así, no puede dejar de subrayarse el papel que la jurisprudencia del TF ha tenido en la progresiva incorporación de garantías en el procedimiento administrativo (fundamentalmente en su desarrollo por parte de los municipios, tal como se acaba de aludir). Las diversas reformas de la Ley de 1952 (la última de ellas entro en vigor en enero de 2009) ya habían incorporado la doctrina garantista del Tribunal:

Así, el art. 15b (ahora, art. 16) exige que toda denegación de naturalización se halle motivada y se base en una solicitud expresa de rechazo; en el mismo sentido debe entenderse la previsión del art. 15c (ahora, art. 17) que establece la serie de datos que han de facilitarse a los electores sobre las personas que se someten al procedimiento de naturalización (procurando que se resguarde al máximo la privacidad del solicitante) en la medida en que se tendrán en cuenta a la hora de decidir el modo de vida y las convicciones que permitan considerar si realmente se ha acomodado al modo de vida suizo (o del concreto Cantón que tramita la solicitud por ser aquel en el que debe demostrarse que se halla integrado) y si no representa riesgo alguno para la seguridad privada o pública.

Por consiguiente, la nueva reforma total de 2014 poco añade, como decíamos, en las cuestiones ya legalmente asumidas por imposición de la doctrina del $\mathrm{TF}^{38}$. Sin embargo, la nueva Ley de 2014 sí que ha operado cambios de carácter orgánico, ya aludidos supra, sobre la mejor coordinación de las autoridades competentes y de búsqueda de la concordancia con la reciente legislación de extranjería; es esta última finalidad la que ha llevado a ampliar las previsiones del actual art. 11 y 12 referidos a los parámetros o criterios con los que se debe valorar la integración de un extranjero.

A la tradicional exigencia de integración que giraba en torno a tres criterios (familiarización con los modos de vida suizos, aceptación de sus valores y no representar riesgo alguno para la seguridad) se añaden ahora otros criterios nuevos:

a) la facultad de expresarse en la vida cotidiana, oralmente y por escrito, en al menos una lengua nacional,

${ }^{38}$ Si acaso, bastaría con recordar el mandato, también dirigido a municipios y cantones, para que se establezcan las correspondientes instancias de recurso (art. 46 de la nueva Ley). 
b) el participar en la vida económica o adquirir una formación y, por último,

c) haber asumido la responsabilidad y sostenimiento de la familia.

Sin embargo, en lo que se refiere a los requisitos formales, en concreto temporales, la exigencia mínima de haber residido en Suiza 12 años $^{39}$ se ha reducido a $10^{40}$ pudiendo contarse como dobles (cada año cuenta como dos) aquellos años «de formación» que hayan transcurrido en el territorio suizo; es decir, cuando en el tiempo vivido en Suiza se tenían entre 8 y 18 años de edad, sin que, en todo caso, la estancia efectiva pueda ser menor de 6 años.

\subsubsection{Naturalización simplificada o «facilitada»}

En segundo lugar, la naturalización también puede lograrse mediante un procedimiento más simplificado en el que, no obstante, siempre se exigen los requisitos que hemos llamado tradicionales (respetar la seguridad y el orden públicos y compartir los valores constitucionales sin que quede comprometida la seguridad interior o exterior del Estado).

Tal procedimiento más ventajoso, o más fácil de superar, se aplica a los casos contemplados en los arts. 21 a 24 de la nueva Ley: al cónyuge de un ciudadano suizo (art. 21); en el caso de que haya de subsanar los casos de ciudadanía reconocida por error (art. 22); para el menor apátrida (art. 23) y, por último, en el caso del hijo de un extranjero naturalizado que no hubiera sido incluido en el expediente de naturalización del padre al haberla solicitado cuando él era menor, cuando este alcanza la mayoría de edad, podrá solicitarla por su cuenta antes de los 21 años (art. 24) y por este procedimiento simplificado.

En estos casos de simplificación o de «vía rápida» (lógicamente, con requisitos menos exigentes ${ }^{41}$ que los previstos para la naturalización ordinaria) solo es competente para conceder la naturalización la Confederación (correspondiendo la decisión a la Oficina federal de inmigración), aunque deberá oír al Cantón afectado.

En definitiva, si bien la nueva Ley ha aligerado algo los plazos de residencia exigidos, no cabe afirmar que se hayan aligerado sus exigencias de integración sino al contrario; con la nueva Ley lo que se trata de garantizar no es tanto un

\footnotetext{
39 Art. 15 de la Ley de 1952.

40 Art. 9 de la Ley de 2014.

${ }^{41}$ El período de residencia en territorio suizo, por ejemplo, es más breve (de 3, 5 o 6 años, según los casos).
} 
largo período de residencia previa sino la real integración en la sociedad suiza; por ello, no cabe desconocer la importancia de sus exigencias que derivan, básicamente del conocimiento de la lengua escrita. De otra parte, la importancia de su tramitación en los niveles locales hasta el punto que no se deba remitir a la Confederación el expediente hasta que se halle aceptado en el nivel municipal y cantonal, generó la preocupación por la excesiva diversidad de plazos que podrían poner en riesgo la seguridad de la tramitación. De ahí que se intente establecer límites por parte de la Confederación; pero su propósito de homogeneizar los plazos desató una reacción en los Cantones (puesta de relieve en el procedimiento de consulta de la propia ley ${ }^{42}$ ).

\section{PARA CONCLUIR: EL CÍRCULO CERRADO DE LA VOLUNTAD POPULAR COMO DECISIÓN ÚLTIMA}

Una de las más curiosas situaciones planteadas en Suiza en relación con los procesos de naturalización se ha producido a raíz de la competencia del pueblo (en el ejercicio de sus derechos políticos) para adoptar decisiones de todo orden (y en todo ámbito territorial) y también las referidas a la naturalización que, al decir de Grisel, es un problema típicamente suizo en el que se entrecruzan principios importantes en los que se pone en juego el equilibrio entre las autoridades y el buen funcionamiento de la democracia ${ }^{43}$.

Justamente por su íntima relación con el ejercicio de los derechos políticos, vale la pena concluir esta aproximación al cuerpo electoral como órgano de decisión, tanto en lo político como en lo jurídico, con una breve referencia a la problemática surgida en torno a la decisión sobre naturalizaciones en Emmen ${ }^{44}$ y Zurigo que provocó la intervención de diversas autoridades y, entre ellas, del Tribunal Federal que se pronunció en sendas sentencias de 2003 (TF129, I, 217 e 129, I, 232), sobre ambos casos respectivamente, en términos que acabaron obligando a llevar a cabo una reforma más en la Ley de ciudadanía.

Las dos sentencias se entrelazan en una argumentación que plantea por vez primera la posibilidad de que un acto político, y discrecional, como es el resul-

${ }^{42}$ V. Mensaje del Consejo Federal sobre la Ley, ya cit., pág.2585.

43 Grisel, Etienne, Le rôle du juge et du législateur dans la question des naturalisations, disponible en red: httpwww.c2d.chadminphpuploadsConf_2003_Geneva_Grisel.pdf.pdf.

44 Ver 3, Droit...ya cit., págs. 43 y ss. Emmen es un municipio de cerca de 27.000 habitantes, en el Cantón de Lucerna, que en el 2000, sobre 57 solicitudes de naturalización (de las cuales 23 eran de niños), todas las cuales habían sido formuladas por ciudadanos de la ex Yugoslavia (39 en total) y fueron rechazadas. 
tado de un referéndum, pueda ser controlado judicialmente; si en el primer caso, anulando la decisión, analiza si existe el derecho a la naturalización y, por consiguiente, su reconocimiento sería un acto administrativo y no, como siempre ha sido considerado, de carácter político; en el segundo llega a poner en tela de juicio la capacidad del cuerpo electoral para pronunciarse sobre tal cuestión respetando debidamente los requisitos jurídicos.

Pero la cuestión aún se complicó más pues la previa determinación del carácter administrativo o político del acto de naturalización enjuiciado comportaba la imposibilidad, o no, de ser revisado por el poder judicial. Grisel critica duramente las dos decisiones por creer que han generado confusión y muchos problemas jurídicos derivados de la anulación del acto recurrido (que afectaba a cientos de disposiciones) mientras que el Tribunal podía haberse limitado a llamar la atención sobre la necesidad de respetar las formas y tratar de evitar las discriminaciones para que los Cantones y los municipios hubieran adaptado progresivamente sus disposiciones.

En el fondo, el Tribunal tomó como punto de partida cierta prelación entre grupos de derechos situando los de carácter individual de los solicitantes de la naturalización sobre el ejercicio de los derechos políticos ${ }^{45}$ de los nacionales suizos, lo que, según Grisel, no puede afirmarse con tal seguridad puesto que la Constitución y las leyes son claras al reconocer tal función a órganos políticos, ya sean de carácter representativo o asambleario. Y, siendo ello así, la cuestión se traslada a la de la capacidad de los Tribunales para modificar la Constitución y las leyes: «en democracia ¿quién es el soberano y tiene, por consiguiente la última palabra, el autor de la Constitución o su intérprete?» ${ }^{46}$. El Tribunal, a juicio del autor, olvida en tales decisiones de 2003 que, en democracia, el pueblo, incluso cuando se equivoca, siempre tiene razón.

45 La cuestión creo que es muy interesante y dudo que entre nosotros hubiera podido llegarse a una decisión de este tipo que requiere de una previa determinación de los grupos de derechos fundamentales y de las eventuales relaciones que entre ellos pueden surgir. Sobre ello me he pronunciado en diversas ocasiones defendiendo soluciones del tipo de las que aquí se aluden y que, en cambio he echado de menos en importantes decisiones del TC español en el que difícilmente cabe encontrar una clara defensa de los derechos individuales (que en alguna ocasión he denominado personalísimos) frente a los derechos que podemos llamar democráticos y que creo deben conocerse como libertades públicas, en especial la de expresión e información. A este respecto, me permito el reenvío a SÁnCHEZ Ferriz, Remedio, Estudio sobre la libertades, Valencia, Tirant lo Blanch, 1996. Idem, Delimitación de las libertades informativas. Valencia, Tirant lo Blanch, 2004. IDEm, Libertades de Reunión y Asociación de los extranjeros. Respuesta del Tribunal Constitucional a la impugnación por diversas Comunidades Autónomas a la Ley 8/2000, en Corts. Anuario de Derecho Parlamentario. 2008, págs. 165-187.

46 Grisel, Etienne, Le rôle du juge et du législateur dans la question... ya cit., pág. 7. 
Dicho esto, debe observarse que aun en la Ley de 2014 el art. 15.2 permite que pueda diferirse a la Asamblea municipal que, en los municipios más pequeños es el órgano legislativo formado por todos los que poseen derecho de voto ${ }^{47}$. En estos casos existe una sustancial identidad entre legislativo y cuerpo electoral pero la garantía para los solicitantes se respeta mediante la obligación de motivar adecuadamente la denegación a través de una moción depositada en la misma Asamblea (art. 16. 2) y por la posibilidad de recurrir sus decisiones ${ }^{48}$.

En conclusión, los derechos de ciudadanía en Suiza son, como hemos visto, un campo específico de confluencia de las principales características del régimen. Si por una parte ellos dan paso a la posibilidad de participar a través de los institutos de democracia directa en las decisiones de las autoridades; de otra, el derecho a acceder a tal status de nacional, por vía de la naturalización, ha de pasar por los tres grados de la nacionalidad ${ }^{49}$ derivados de la regulación federal, cantonal y municipal que se superponen dando lugar a una triple intervención o análisis de cada caso concreto. Las decisiones del Tribunal federal que se acaban de comentar no modifican la función ni significación de los derechos políticos pero sin duda sí han logrado introducir en punto a la naturalización algún elemento garantista que se refleja en la nueva Ley de ciudadanía.

\section{BIBLIOGRAFÍA}

AuberT, Jean-François. Exposé des institutions politiques de la Suisse à partir de quelques affaires controversées. Lausanne, Payot, 1978.

Aubert, Jean-François y MAHon, Pascal, Petit commentaire de la Constitution fédéral de la Confédération suisse du 18 avril 1999. Zurich, Schulthess, 2003.

Auer, Andreas, Malinverni, Giorgio y HotTelier, Michel, Droit constitutionnel suisse. Berne, Stämpfli Editions SA, 2006.

Auer, Andreas. Le caratteristiche della democrazia diretta locale in Svizzera, en Amministrare, 1999.

Fleiner, Thomas, Misic, Alexander, Töpperwien, Nicole. Suiss Constitutional Law, The Hague, Kluwer Law International, 2005.

${ }^{47}$ El mapa municipal suizo cuenta con multitude de casos en los que el municipio se rige por decisiones adoptadas en asamblea popular y aplicadas por el órgano ejecutivo. Por todos, AUER, Andreas. Le caratteristiche della democrazia diretta locale ... ya cit.

48 Gerottro, Sergio, Suiza..., op. cit., págs. 110 y ss. El extraordinario interés de tan complejo sistema y la claridad con que el autor citado lo sintetiza me llevan a recomendar la lectura de las págs. 110 a 114 .

49 Auer, Andreas, Malinverni, Giorgio, y Hottelier, Michel, Droit constitutionnel suisse... ya cit., pág. 127. 
FLEINER, Thomas. Le federalisme suisse. Les principes constitutionnels du fédéralisme de type multiculturel, en MAZzOLLENI, Oscar (a cura di) Federalismo e decentramentol Fédéralisme et décentralisation. L'esperinza svizzera e le nuove sfide europeel L'expérience suisse et les nouveaux défis européens. Milano, Giampiero Casagrande, 2005, págs. 27-41.

FLEINER, Thomas. «The current situation of federalism in Switzeland», en Revista d'Estudis Autonomics I Federals, núm. 9, 2009, págs. 51 a 90.

FLORIDIA, Giorgio, Profili di tecnica redazionale dell' «aggiornamento» della costituzione svizzera (ovvero: la forma è sostanza), in Reposo, Antonio (a cura di), La revisione della Costituzione federale svizzera, Giappichelli, Torino, 2000.

Fossedal, Gregory A. Direct Democracy in Switzerland. Transaction publishers, 2007.

Gerotto, Sergio, Suiza. Sobre cómo se gobiernan los suizos. Madrid, CEPC, 2015.

— La «dinámica costituzionale» nella Confederacione svizzera: qualche spunto di riflessione sul ruolo dei diritti popolari nel procedimento di revisione costituzionale, en Il Diritto della Regione, (Liber amicorum per Nino Olivetti Rason), num. 5-6 2009, págs. 119 y ss.

GrISEL, Etienne, Initiative et referéndum populaires. Traité de democratie semi-directe en droit Suisse. Bern, Stämpfli Editions, 2004.

- Le rôle du juge et du législateur dans la question des naturalisations, httpwww.c2d. chadminphpuploadsConf_2003_Geneva_Grisel.pdf.pdf

JARIA Y MANZANO, Jordi. «El federalismo suizo. Una respuesta a una sociedad plural». RVAP, núm. 61, 2001, págs. 89 y ss.

KNAPP, Blaise. L'Ordinamento federale svizzero (con saggio introduttivo di Giovanni Guiglia) Torino, Giappichelli. 1994.

Koller, H. y Biagnni, G.: «La nueva Constitución federal suiza. Una visión general de las novedades y los aspectos más destacados», en Teoría y Realidad Constitucional, núm. 10-11, 2. ${ }^{\circ}$ semestre de 2002 y primer semestre de 2003, pp. 611 y ss.

LiNDER, Wolf. Fédéralisme Suisse et culture politique, en MAZzolleni, Oscar (a cura di) Federalismo e decentramento/Fédéralisme et décentralisation. L'esperinza svizzera e le nuove sfide europeel L'expérience suisse et les nouveaux défis européens. Milano, Giampiero Casagrande, 2005, págs. 43-59.

Le Roy, Ives y Schoenenberger, Introduction générale au droit Suisse. LGDJ 2008. LinDSAY, Katherine Federal constitutional law. Lawbook co. 2003.

Mahon, Pascal. Droit constitutionnel. 3 ed. Helbing Lichtenhahn, Vol. I, 2015.

Martenet, Vincent. L'Autonomie constitutionnel des Cantons. Bâle-Geneve, Helbing\&Lichtenhahn, 1999.

Papadopoulos, Yannis. «Les mécanismes du vote référendaire en Suisse: l'impact de l'offre politique». In Revue française de sociologie. 1996, 37-1. pp. 5-35. http:// 
www.persee.fr/web/revues/home/prescript/article/rfsoc_0035-2969_1996_ num_37_1_7078.

SÁnCHEz FERriz, Remedio y GARCía Soriano, María Vicenta. Suiza (Una realidad bistórica para el Siglo XXI). Madrid, CEPC, 2001.

SÁNCHEZ FERrIZ, Remedio. «Reflexiones sobre una vieja experiencia descentralizada: siglos de convivencia entre todo tipo de diversidades en el corazón de Europa», en Revista Valenciana d'Estudis Autonòmics, núm. 59, 2014, Vol. I, págs. 5-27.

— «Un mecanismo de integración federal y ciudadana: las consultas "prenormativas” del ordenamiento constitucional suizo, en Teoría y Realidad Constitucional, núm. 36, 2015, pp. 353-376.

SIGG, Oswald. La Svizzera política. Zurigo, Pro Helvetia, 1996.

Thalmann, Urs. Derechos constitucionales y federalismo: el caso de Suiza, en Aparicio, Miguel Ángel, Derechos y libertades en los Estados compuestos. Barcelona, Atellier, 2005, págs. 101 y ss.

Title:

The Swiss people: Their constitutional significance and composition.

\section{Summary:}

The people (electorate) as the supreme organ in all levels of political decision-making. 2. Composition of the electoral body. Citizenship and political rights. 3. To conclude: the closed circle of the popular will as a final decision.

\section{Resumen:}

El pueblo, que se expresa como cuerpo electoral (o conjunto de ciudadanos que tras la mayoría de edad pueden ejercer los derechos políticos), es, elemento constitutivo y constituyente de la Confederación (tanto en su dimensión federal como en la cantonal) y, a la vez, órgano supremo y primera fuente de legitimidad del ordenamiento constitucional suizo. El pueblo participa en las decisiones que comportan incorporaciones al mismo dando lugar en ocasiones a conflictos con las decisiones de las autoridades competentes. 


\begin{abstract}
:
The people who are defined as the electorate (the citizens who after the age of majority exercise in reality their political rights), are the constitutive and constituent element of the Confederation (both at federal and cantonal level) and, simultaneously, the supreme organ and the principal source of legitimacy in the Swiss constitutional order. The people participate in decisions that involve additions to them some time generating serious conflicts with the competent authorities.
\end{abstract}

Palabras clave:

Poder constituyente. Órgano supremo del Estado.

Keys words:

Constituent power. The supreme organ of the state. 
